\title{
Interlocking friction governs the mechanical fracture of bilayer $\mathrm{MoS}_{2}$
}

Gang Seob Jung ${ }^{1 *}$, Shanshan Wang ${ }^{2 *}$, Zhao Qin ${ }^{1}$, Francisco J. Martin-Martinez ${ }^{1}$, Jamie H. Warner ${ }^{2 \dagger}$, and Markus J. Buehler ${ }^{1,3 \dagger}$

\footnotetext{
${ }^{1}$ Laboratory for Atomistic and Molecular Mechanics (LAMM), Department of Civil and Environmental Engineering, Massachusetts Institute of Technology, 77 Massachusetts Ave., Cambridge 02139, MA, USA
}

${ }^{2}$ Department of Materials, University of Oxford, Parks Road, Oxford, OX1 3PH, United Kingdom

${ }^{3}$ Center for Computational Engineering, Massachusetts Institute of Technology, 77 Massachusetts Ave., Cambridge, MA 02139, USA

†Email: (jamie.warner@materials.ox.ac.uk) or (mbuehler@mit.edu).

*These authors contributed equally to this work

\begin{abstract}
Molybdenum disulfide $\left(\mathrm{MoS}_{2}\right)$ monolayer is a two-dimensional (2D) material, which is expected to provide the next generation of electronic devices together with graphene and other nanomaterials. Due to its significance for future electronics applications, gaining a deep insight of the fundamental mechanisms upon $\mathrm{MoS}_{2}$ fracture is crucial to prevent mechanical failure towards reliable applications. Here, we report direct experimental observation and atomistic modeling of the complex failure behaviors of bilayer $\mathrm{MoS}_{2}$, revealing highly variant interlayer frictions, elucidated with in-situ transmission electron microscopy and large-scale reactive molecular dynamics simulations. Our results provide systematic understanding of the effects that different stacking and loading conditions cause on the failure mechanisms and crack-tip behaviors in bilayer $\mathrm{MoS}_{2}$ systems. Our findings unveil essential properties in fracture of this $2 \mathrm{D}$ material and provide mechanistic insight into its mechanical failure.
\end{abstract}

Keywords: $\mathrm{MoS}_{2}$, van der Waals bilayer, crack propagation, two-dimensional material, insitu TEM

\section{Submitted to: ACS Nano}

Fundamental fracture mechanics is of crucial concern for materials engineering and subsequent applications, which requires understanding the underlying mechanisms governing crack propagation in broad range of scientific disciplines. ${ }^{1-3}$ As a post-graphene material, molybdenum disulfide $\left(\mathrm{MoS}_{2}\right)$ monolayer is a two-dimensional (2D) material from the transition metal dichalcogenide (TMD) family that has attracted considerable attention due to its remarkable electronic and optoelectronic properites. ${ }^{4-7}$ These $2 \mathrm{D}$ materials show distinct mechanical behaviors ${ }^{8}$ and their atomically thin structures provide opportunities for observing atom dynamics ${ }^{9-10}$ and manipulating atomic configurations. ${ }^{11}$ Differently from bulk materials, a single bond breaking in these $2 \mathrm{D}$ structures can result in crack propagation due to its atomic thinness. Therefore, the behavior of crack-tip in 2D materials is strongly affected by atomic scale features near the crack-tip, e.g., defects and grain boundaries. ${ }^{12-13}$

Furthermore, there are emerging applications and fundamental engineering arising from the addition of more layers to the system, e.g., electro-mechanics ${ }^{14-16}$ and van der Waals (vdW) heterostructures, ${ }^{17-19}$ where the interlayer interaction becomes more crucial for their fracture. 
Thus, characterizing interlayer interactions and understanding their roles in the mechanical stability and failure are of great importance for the development of devices based on the combination of 2D materials. To understand the multilayer 2D systems, the stacking geometries and binding energies of differently stacked bilayer $\mathrm{MoS}_{2}$ systems have been reported. ${ }^{20-21}$ Despite these previous initiatives, the atomic-scale mechanics of crack-tip behaviors in multilayer $\mathrm{MoS}_{2}$ systems have been elusive so far due to challenges associated to its direct experimental observation and development of predictive atomic-scale models. Specifically, it is quite elusive how the interlayer interaction of $\mathrm{MoS}_{2}$ may interplay with the crack-tip behaviors in one of the layers.

Our previous study has demonstrated that in-situ aberration-corrected transmission electron microscopy (AC-TEM) and molecular dynamics (MD) simulations are efficient tools to investigate the dynamics of crack-tip behaviors and fracture of $\mathrm{MoS}_{2}$ monolayer. ${ }^{12} \mathrm{By}$ utilizing these tools, we report here the dynamics of crack propagation in suspended bilayer $\mathrm{MoS}_{2}$ and address questions related to the effects of the uncracked layer on crack propagation in the cracked layer. Our finding reveals atomic-scale features and interlayer interactions of the multilayer system with 2D materials, which impacts the bottom-up design of vdW heterostructures and applications of electro-mechanics.

\section{RESULTS AND DISCUSSION}

One of the most promising methods to produce high-quality and large-area $\mathrm{MoS}_{2}$ is chemical vapor deposition (CVD). By adjusting the synthesis parameters properly, the $\mathrm{MoS}_{2}$ samples contain regions of both monolayer and bilayer. In our experimental setup, cracks are introduced into $\mathrm{MoS}_{2}$ by popping a strained $\mathrm{MoS}_{2}$ membrane with a focused high-energy electron beam (Figure 1a, See Methods) to cause fractures that enable the observation of the crack-tip behaviors with AC-TEM. The cracks that initially appear in monolayer regions then propagate into bilayer domains in Figure $1 \mathrm{~b}$ (i-iv). The single atom resolution of AC-TEM and the application of Fast Fourier Transformation (FFT) to TEM images (See Methods), enables the extraction of specific lattice information in the reciprocal space to successfully reconstruct the atomic details of each layer in the bilayer $\mathrm{MoS}_{2}$ as demonstrated in Figure $1 b(v-v i i i)$.

To obtain a reliable forcefield for performing MD simulations that are able to reproduce the behavior of crack-tips in bilayer $\mathrm{MoS}_{2}$ system, we adapted the reactive forcefield (REBO) parameters by using dispersion-corrected DFT (Density Functional Theory) calculations to describe well both mechanical properties of intralayer and interlayer interactions of $\mathrm{MoS}_{2}$ (See SI Methods 1-3). As shown in Figure 1c, the crack behavior with bilayer region is well captured in Figure 1c. Then, the subsequent MD simulations, allow us to systematically measure the friction between two layers with different stacks and loading directions by using a circular top layer. This is shown in Figure 2a (See SI Method 4). The magnitude of the friction significantly depends on the stacking and loading conditions (Figure $2 b$ ). The results show that frictions in \pm armchair (AC) directions with $2 \mathrm{H}$ and 3R stacks can reach 10 times higher than turbostratically-stacked bilayer systems. The origin of difference for $2 \mathrm{H}$ and $3 \mathrm{R}$ with respect to turbostratically-stacked systems are expected to come from different binding energies as shown in Table S1 and S2. However, the binding energies of $2 \mathrm{H}$ or $3 \mathrm{R}$ stacking are only $\sim 1.5$ times larger than those of AA stacking. Instead, analyzing the relative position of sulfur atoms in top and bottom layers indicate geometric effects on the friction. As shown in Figure 2c, sulfur positions in \pm zigzag (ZZ) directions are highly symmetric while sulfur positions in $\pm \mathrm{AC}$ directions are asymmetric. In fact, the peaks of friction forces occur when 
the sulfur atoms of the top layer align to the sulfur atoms of the bottom layer. Our results show that the slopes are equal to all peaks due to the geometry and number of interacting sulfur atoms per unit length. Therefore, we conclude that such large difference result not from the vdW interaction itself but geometrical interlocking between two layers.

Also, we found that the friction per unit area of stacked bilayer is likely to decrease as the stacked areas increase (See Tables S4 - S8) because local ripples facilitate the sliding as the stacked areas increase. Also, we have evaluated the loading rate dependence. The results show that the friction values are likely to decrease as the loading rate decrease as shown in Figure S3. However, the friction values of $2 \mathrm{H}$ and $3 \mathrm{R}$ systems are less sensitive to the loading rate compared to turbostratically-stacked layers, which means that the friction from wellstacked layers can be much stronger than the friction coming from turbostratically-stacked layers at the slow loading rate limit. These unique geometrically interlocking friction mechanisms of $\mathrm{MoS}_{2}$ allow us to understand complicated crack-tip behaviors observed with in-situ AC-TEM.

\section{Crack tips in turbostratically-stacked bilayer $\mathrm{MoS}_{2}$ region: crack blocking}

Figure 3 shows the experimental observation of crack propagation in turbostratically-stacked bilayer regions with AC-TEM, together with MD results. In turbostratically-stacked systems one of the layers can be removed in AC-TEM images by using a mask in Fourier space to study the crack in detail without the interference of the second layer. The AC-TEM images reconstructed by these means show the atomic structures of both the cracked and uncracked regions separately, which can be directly compared to the simulations results. Both AC-TEM images and MD indicate that the crack prefers the zigzag direction for propagating, and as a consequence the sliding of the cracked layer with respect to the uncracked one generate different moiré pattern in Figure $3 \mathrm{a}$ and $\mathrm{d}$.

To see how the friction from the different stacking can disturb the crack propagation, we also performed MD simulations with various angles of stacked conditions (See Crack blocking in Methods and Figure S3a). Due to the elastic energy, a crack naturally propagates into the circular bilayer regions. The propagation of the crack is disturbed drastically and finally stops at the center of circular region with $0^{\circ}(3 \mathrm{R})$ and $60^{\circ}(2 \mathrm{H})$. However, other stacking angles (e.g., $15^{\circ}, 30^{\circ}$, and $45^{\circ}$ ) cannot disturb cracks enough as shown in Figure S3b (See Supporting movies 1 and 2 for $15^{\circ}$ and $2 \mathrm{H}$ ). Additionally, Figure 3 shows the good agreement between the moire patterns that appear during the crack propagation with $15^{\circ}$ rotated turbostratic stack from experimental observation and MD simulations. The asymmetric moiré patterns along the crack result from asymmetric friction from the other layer.

\section{Crack propagation through the entire bilayer region: coherent fracture}

Figure 4 shows two distinct fracture behaviors in suspended $2 \mathrm{H}$-stacked $\mathrm{MoS}_{2}$ that are generated by puncturing the membrane with the electron beam irradiation in a monolayer region. The crack on the left propagated too fast to be captured with the AC-TEM imaging technique, while the crack on the right propagated slowly enough to be tracked. Despite the fast propagation of the first crack, the image shows a complete fracturing of both layers, while the slow crack is confined in one layer without any interruption of the second layer. Although friction force from fast crack propagation should be higher than that from slow propagation, this loading rate dependence is not sufficient to explain the coherent fracture.

To test whether the uncracked layer breaks due to the crack propagation in the second layer, we performed MD simulations of a $30 \times 40 \mathrm{~nm}$ rectangular shape bottom layer with an $8 \mathrm{~nm}$ length initial crack. To evaluate the differences coming from stacking conditions, $30 \times 30 \mathrm{~nm}$ 
square-shaped top layer is stacked with $0^{\circ}(3 \mathrm{R}), 60^{\circ}(2 \mathrm{H})$, and $15^{\circ}$. The top layer is assumed to be semi-infinite with periodic boundary conditions (See Coherent fracture in Methods and Figure 7a). We found that the top layer does not break even if the pre-strain increases in both layers, which corresponds to faster propagation due to higher elastic energy.

The crack propagations cause local ripples in the cracked layer and it disturbed the coherent behaviors (consisting $2 \mathrm{H}$ stack) as shown in Figure 4e, reducing the friction significantly. Once the $2 \mathrm{H}$ stacking is distorted, it does not recover its alignment due to the mismatching strains at both layers. However, this behavior changes drastically if the top layer has a small flaw on the crack propagation path. Due to this flaw, the regions near the flaw are not well stacked in $2 \mathrm{H}$ arrangement anymore before the crack path, allowing coherent movement cause effective tensile stress near the crack tip after first crack passed as shown in Figure $4 \mathrm{f}$. Therefore, the small flaw becomes a crack-tip following the first crack path. It is important to note that this flaw is too small to break along itself. Also, the coherent fracture happens with 3R, but not with turbostratic stacks with the same conditions, as shown in Figure S6b (See Supporting movies 3 and 4 for $15^{\circ}$ and $2 \mathrm{H}$ stacks with a $2 \mathrm{~nm}$ flaw).

\section{Cracks in $2 \mathrm{H}$ stacked bilayer $\mathrm{MoS}_{2}$ : crack branching}

Figure 5a shows a region in the bilayer $\mathrm{MoS}_{2}$ where the $1^{\text {st }}$ crack tip is in the monolayer region and the $2^{\text {nd }}$ crack goes through both layers. There is lattice distortion along the $1^{\text {st }}$ crack path, which could be a result from defects and grain boundaries. As shown in the Figure $5 \mathrm{e}$, moire patterns are observed at the front of the $1^{\text {st }}$ crack. Although these irregular moiré patterns could be thought to arise from a local ripple of one layer, the remaining tensile strain of the other layer prevents the local ripple to appear with such extremely confined width and non-straight shape ${ }^{22}$. Also, if this comes from grain boundaries, good $2 \mathrm{H}$-stacking alignment in other regions is not well explained. Thus, it is reasonable to conclude that the distortion comes from defects. These unique structures can happen when two $2 \mathrm{H}$-stacked separated triangles on monolayer $\mathrm{MoS}_{2}$ combine during CVD growth-process. Furthermore, the $1^{\text {st }}$ crack path is on the defected region of the $2^{\text {nd }}$ layer, which can result from weakening friction in the non-coherent $2 \mathrm{H}$-stacked regions. Surprisingly, the $1^{\text {st }}$ crack branches into highly complicated paths (Figure S8), in contrast to other observed cracks.

To understand this complex crack-tip behavior in the system, we simplified the model with some critical features. First, the entire system is considered as a $2 \mathrm{H}$-stacked bilayer $\mathrm{MoS}_{2}$. Second, A thin defected region is prepared in the uncracked $2^{\text {nd }}$ layer. Finally, the system is constrained not only in the direction perpendicular to the crack propagation but also in the crack propagation direction, which implies fixing all boundaries. Within these settings, we simulated a $40 \times 40 \mathrm{~nm}$ bilayer system with an initial crack inserted in the $1^{\text {st }}$ layer. The $2^{\text {nd }}$ layer has 2nm-width defect regions with different defect ratios, as shown in Figure S7a (See Crack branching in Methods). As the defect ratio increases, the crack path becomes more disturbed and eventually branches when $10 \%$ defects ratio is reached (Figure S7 and Figure $5 \mathrm{i}$ and Supporting movies 5 and 6 for $0 \%$ and $10 \%$ ).

Branched directions of the crack also show the same trend with AC-TEM images. One crack is likely to pass straight following a zigzag edge direction, and the other crack is deflected to the armchair edge direction. The asymmetry in the friction forces due to non $2 \mathrm{H}$-stacking controls branched directions shown in Figure 5i. The branching points in AC-TEM images show non $2 \mathrm{H}$-stacked moiré patterns, as well as the same branching directions from MD simulations. While one crack propagates straight, the other one deflects, as shown in Figure 5 and Figure S8. In AC-TEM images, the branched cracks run parallel and leave islands behind as shown in Figure S8, which is not mechanically driven without sliding but induced by 
electron beams because the islands become smaller by losing atoms without moiré pattern changes.

\section{CONCLUSION}

We report that bilayer $\mathrm{MoS}_{2}$ has unique interlayer friction between the layers that depend not only on the stacking conditions but also on the loading conditions. Especially, 2H and 3R stacking arrangements show extremely high static friction compared to turbostraticallystacked bilayers due to their geometrical interlocking coming from sulfur-to-sulfur positions. This is the first report of the geometrical interlocking mechanism in bilayer $\mathrm{MoS}_{2}$. Also, we observed various crack-tip behaviors in bilayer $\mathrm{MoS}_{2}$ system: 1) a crack can either propagate or be blocked due to the different frictions. 2) a crack in the first layer can induce breaking of the other layer. 3) a crack in the first layer branches due the existence of defects or atomic configuration in the other layer. The observed complex behaviors can be explained by the principles we derived:

- High friction from sulfur-to-sulfur interlocking of $2 \mathrm{H}$ and $3 \mathrm{R}$ stacks

- Low friction of turbostratic stacks

- Loading-directional dependence of slip-stick frictions

These essential insights into complex fracture of bilayer $\mathrm{MoS}_{2}$, can also apply to other transition metal dichalcogenide (TMD) materials, which further increases the relevance of this scientific contribution.

\section{METHODS}

\section{CVD synthesis and transfer of $\mathrm{MoS}_{2}$ sample}

$\mathrm{MoS}_{2}$ monolayers and bilayers were prepared using a similar approach to that previously reported. ${ }^{23}$ Molybdenum trioxide $\left(\mathrm{MoO}_{3}, \geq 99.5 \%\right.$, Sigma-Aldrich) and sulphur (S, $\geq 99.5 \%$, Sigma-Aldrich) powder were used to grow monolayer $\mathrm{MoS}_{2}$ on a $\mathrm{SiO}_{2} / \mathrm{Si}$ substrate $(300 \mathrm{~nm}$ thick $\mathrm{SiO}_{2}$ ) by chemical vapor deposition (CVD) at atmospheric pressure. Two furnaces were used to enable temperature control on both the precursors and the substrate. The heating temperatures for $\mathrm{S}, \mathrm{MoO}_{3}$ and $\mathrm{SiO}_{2} / \mathrm{Si}$ substrate were $\sim 180, \sim 300$, and $\sim 800{ }^{\circ} \mathrm{C}$, respectively, with argon used as the carrier gas. To avoid cross-contamination between $\mathrm{MoO}_{3}$ and $\mathrm{S}, \mathrm{MoO}_{3}$ powder was placed in an inner tube having a smaller diameter, which was then inserted into the larger one-inch quartz tube. The S powder was loaded in the outer quartz tube. The substrate was oriented vertically to increase the uniform growth area of $\mathrm{MoS}_{2}$ film. This CVD method grew continuous monolayer $\mathrm{MoS}_{2}$ films with $\sim 10 \%$ of the area covered by bilayers. The as-grown product was checked under the optical microscope and the regions having a high percentage of bilayer $\mathrm{MoS}_{2}$ were marked for the following transfer procedure. $\mathrm{MoS}_{2}$ was transferred onto the $\mathrm{Si}_{3} \mathrm{~N}_{4}$ TEM grid (Agar Scientific AG21580) via a standard polymerbased method. The surface of the $\mathrm{MoS}_{2} / \mathrm{SiO}_{2} / \mathrm{Si}$ substrate was first spin-coated with a thin film of poly (methyl methacrylate) (PMMA) followed by floating it on a $1 \mathrm{M}$ potassium hydroxide $(\mathrm{KOH})$ solution to etch the $\mathrm{SiO}_{2}$ away. After the PMMA/MoS $\mathrm{P}_{2}$ film was detached from the Si substrate, the film was rinsed in deionized water for several times. Subsequently, the regions in the film with a high percentage of bilayer $\mathrm{MoS}_{2}$ were scooped up using a holey $\mathrm{Si}_{3} \mathrm{~N}_{4}$ grid. The grid was dried overnight in air followed by baking at $180{ }^{\circ} \mathrm{C}$ for 15 minutes. Finally, the grid was submerged in acetone for 8 hours to remove PMMA. 


\section{Transmission electron microscopy and TEM data processing}

High-resolution TEM imaging was conducted using Oxford's JEOL JEM-2200MCO field emission gun transmission electron microscope with a CEOS imaging aberration corrector under an accelerating voltage of $80 \mathrm{kV}$. AC-TEM images were recorded using a Gatan Ultrascan $4 \mathrm{k} \times 4 \mathrm{k}$ CCD camera (frame speed: $\sim 25 \mathrm{fps}$ ) with 1-2 s acquisition time. We took one frame approximately every 10-20s in our AC-TEM imaging depending on the speed of the crack propagation and the imaging condition adjustment. Images were processed using the software of ImageJ. They were initially adjusted with a band-pass filter (between 100 and 1 pixel) to modify the long-range non-uniformity on the illumination intensity, and then smoothed by applying a Gaussian blur (2 pixels). For turbostratically assembled bilayer $\mathrm{MoS}_{2}$ with a twist angle except integral multiple of $60^{\circ}$, the lattice configuration corresponding to each layer can be separately reconstructed. It was realized by first doing the FFT of the ACTEM image followed by applying a mask to the 2D FFT image to only choose reflexes contributed by one layer of the bilayer $\mathrm{MoS}_{2}$ in the reciprocal space. Finally, do the inverse FFT to reconstruct the lattice structure of this layer in the real space. Cracks were generated by illuminating suspended $\mathrm{MoS}_{2}$ to the focused electron beam under $80 \mathrm{kV}$, which is similar to prior work ${ }^{12}$. The crack emanated from either a bilayer region or a monolayer area, which can subsequently move into bilayer domains. The generation of the fast fracture in $\mathrm{MoS}_{2}$ by a focused electron beam at the initial stage was due to a homogeneous in-plane tension in suspended $\mathrm{MoS}_{2}$ introduced by the baking process during transfer, which is similar as popping a tight drumhead by a sharp needle. The crack continuously propagated by a proper dose of the electron beam irradiation on $\mathrm{MoS}_{2}$ during imaging, as the electron beam produced a tiny force at the crack tip due to the formation of line defects along fractured edges and the curling up of the freshly cracked edges.

\section{Molecular dynamics simulations}

Molecular dynamics simulations in this study were performed via LAMMPS package. ${ }^{24}$ Reactive Empirical Bond Order (REBO) forcefield (FF) ${ }^{25-27}$ is utilized for the interatomic potential. We obtained the binding energy as a function of distance between two layer from our DFT calculations with DFT-D2 correction, ${ }^{28}$ and utilized the data to optimize the previous parameters. ${ }^{12,29}$ The obtained binding energy and equilibrium distances for $2 \mathrm{H}$ (AA'), 3R(AB), and AA stacks show good agreement with previous DFT studies. ${ }^{21,30} \mathrm{We}$ tuned REBO parameters for both the interatomic interaction and $\mathrm{vdW}$ interaction, which successfully described monolayer stress-strain curves, elastic constants, and bilayer interactions from DFT calculations (See SI Methods). Based on this optimized FF, we performed simulations to measure the friction forces between two layers with different stack conditions, loading directions, and loading rates. We utilized Steered Molecular Dynamics $(\mathrm{SMD})^{31}$ to pull the top layer in the zigzag and armchair directions, allowing natural deformation and localized sliding under various loadings, where the method is generally utilized for deformation of complex biomaterials ${ }^{32}$ (See SI Methods for more details).

\section{Crack blocking: Crack propagation into stacked finite bilayer regions}

We prepared a 30x50nm rectangular $\mathrm{MoS}_{2}$ layer (bottom layer) for the crack region with a circular layer (top layer) with a $14 \mathrm{~nm}$ radius for the uncracked region as shown in Figure S4a. The circular regions are stacked in six different ways: $0^{\circ}(3 \mathrm{R}), 15^{\circ}, 30^{\circ}, 45^{\circ}$, and $60^{\circ}(2 \mathrm{H})$ rotated. In the beginning, the systems are stretched with $3.2 \%$ tensile strain in the $x$ direction, which allows crack propagation and breaks entire layer without the circular layer as shown in Figure S4b. After energy minimization, the system is relaxed with NVT ensemble at low temperature $10 \mathrm{~K}$ for $100 \mathrm{ps}$ with $1 \mathrm{fs}$ time step. We note that the bottom layer is still pre- 
stretched but the top layer is fully relaxed without rotation. Then, NVE ensemble is applied to ignore the undesired effects from thermostat before a crack inserted in the bottom layer. This process helps us to correctly capture the subtle differences from different stacks. After a sharp crack $\left(l_{\mathrm{c}}=15 \mathrm{~nm}\right)$ is inserted in the bottom layer and the crack propagation is observed as shown in Figure $\mathrm{S} 4 \mathrm{~b}$.

\section{Coherent fracture: Crack propagation into stacked semi-infinite bilayer regions}

Instead of finite circular layer, a semi-infinite top layer that is large enough to be prestretched with the bottom layer is modeled. A square layer $(30 \times 30 \mathrm{~nm})$ is stacked on the bottom layer with different angles: $0^{\circ}(3 \mathrm{R}), 15^{\circ}$, and $60^{\circ}(2 \mathrm{H})$. The bottom layer is $30 \mathrm{x} 40 \mathrm{~nm}$ and the major crack length $\left(l_{\mathrm{c}}=8 \mathrm{~nm}\right)$ in the bottom layer is set to $8 \mathrm{~nm}$. Different flaws are introduced in the top layer from 0 to $3 \mathrm{~nm}$ crack $\left(l_{\mathrm{c} 2}\right)$ on the crack-path in the bottom layer as shown in Figure S6a. The flaw lengths are too short to allow the crack propagation in the top layer with the applied pre-strain $(\sim 5 \%)$.

\section{Crack branching: Cracks in $2 \mathrm{H}$ stacked bilayer $\mathrm{MoS}_{2}$}

From the experimental observation in Figure 5, we prepared the $40 \mathrm{x} 40 \mathrm{~nm}$ square layer for the top and bottom layer with $2 \mathrm{H}$-stack as shown in Figure S7a. First, different ratios of defects are introduced in the defect region of top layer where the width is $2 \mathrm{~nm}$ and the length is $40 \mathrm{~nm}$. Each model is relaxed with $4 \%$ pre-strain in the $x$ direction. After energy minimization, the system is relaxed with NVT ensemble at $10 \mathrm{~K}$ for $100 \mathrm{ps}$. The ensemble is changed from NVT to NVE and a crack $(l \mathrm{c} \sim 8 \mathrm{~nm})$ is introduced in the bottom layer. The stored strain energy allows the crack start to propagate, and the natural behaviors of both layers are observed. The left and right boundaries are fixed in the $x$ direction and top and bottom boundaries are fixed in the $y$ direction with non-periodic boundary condition, mimicking the main features from the experimental structure in Figure 5.

\section{Supporting Information}

SI Methods

1. DFT calculation for binding energy of $\mathrm{MoS}_{2}$ bilayer

2. Parameter optimization for vdW binding energy (Reactive FF, REBO)

3. Parameter optimization for mechanical properties of monolayer $\mathrm{MoS}_{2}$ (Reactive FF, REBO)

4. Interlocking friction force: MD simulations

Supporting Tables 1: DFT calculations for binding energy

Supporting Tables 2: DFT calculations for geometry

Supporting Tables 3: Tuned LJ parameters of current forcefield

Supporting Tables 4: Geometric parameters and elastic constants

Supporting Tables 5-8: The values of interlayer frictions

Supporting Figures 1-8

Supporting Movies 1-6

\section{ACKNOWLEDGEMENTS}

Funding and resources: The authors GS.J, Z.Q., F.J M-M and M.J.B acknowledge support by the Office of Naval Research (Grant No. N00014-16-1-233) and DOD-MURI (Grant No. FA9550-15-1-0514). We acknowledge support for supercomputing resources from the Supercomputing Center/KISTI (KSC-2017-C2-0013).

Author contributions: All the authors contributed to the current work and writing the paper. 
Competing interests: The authors declare that they have no competing interests. Data and materials availability: All data needed to evaluate the conclusions in the paper are present in the paper and/or the Supporting Information. Additional data related to this paper may be requested from the authors.

\section{REFERENCES}

(1) Buehler, M. J.; Gao, H., Dynamical fracture instabilities due to local hyperelasticity at crack tips. Nature 2006, 439 (7074), 307-310.

(2) Kermode, J. R.; Albaret, T.; Sherman, D.; Bernstein, N.; Gumbsch, P.; Payne, M. C.; Csanyi, G.; De Vita, A., Low-speed fracture instabilities in a brittle crystal. Nature 2008, 455 (7217), 1224-1227.

(3) Livne, A.; Bouchbinder, E.; Svetlizky, I.; Fineberg, J., The Near-Tip Fields of Fast Cracks. Science 2010, 327 (5971), 1359.

(4) Wang, Q. H.; Kalantar-Zadeh, K.; Kis, A.; Coleman, J. N.; Strano, M. S., Electronics and optoelectronics of two-dimensional transition metal dichalcogenides. Nat Nano 2012, 7 (11), 699712.

(5) Jariwala, D.; Sangwan, V. K.; Lauhon, L. J.; Marks, T. J.; Hersam, M. C., Emerging Device Applications for Semiconducting Two-Dimensional Transition Metal Dichalcogenides. ACS Nano 2014, 8 (2), 1102-1120.

(6) RadisavljevicB; RadenovicA; BrivioJ; GiacomettiV; KisA, Single-layer MoS2 transistors. Nat Nano 2011, 6 (3), 147-150.

(7) Splendiani, A.; Sun, L.; Zhang, Y.; Li, T.; Kim, J.; Chim, C.-Y.; Galli, G.; Wang, F., Emerging Photoluminescence in Monolayer MoS2. Nano Lett. 2010, 10 (4), 1271-1275.

(8) Lee, C.; Li, Q.; Kalb, W.; Liu, X.-Z.; Berger, H.; Carpick, R. W.; Hone, J., Frictional Characteristics of Atomically Thin Sheets. Science 2010, 328 (5974), 76.

(9) Warner, J. H.; Rümmeli, M. H.; Ge, L.; Gemming, T.; Montanari, B.; Harrison, N. M.; Büchner, B.; Briggs, G. A. D., Structural transformations in graphene studied with high spatial and temporal resolution. 2009, 4, 500.

(10) Warner, J. H.; Margine, E. R.; Mukai, M.; Robertson, A. W.; Giustino, F.; Kirkland, A. I., Dislocation-Driven Deformations in Graphene. Science 2012, 337 (6091), 209.

(11) Robertson, A. W.; Allen, C. S.; Wu, Y. A.; He, K.; Olivier, J.; Neethling, J.; Kirkland, A. I.; Warner, J. H., Spatial control of defect creation in graphene at the nanoscale. 2012, 3, 1144.

(12) Wang, S.; Qin, Z.; Jung, G. S.; Martin-Martinez, F. J.; Zhang, K.; Buehler, M. J.; Warner, J. H., Atomically Sharp Crack Tips in Monolayer MoS2 and Their Enhanced Toughness by Vacancy Defects. ACS Nano 2016, 10 (11), 9831-9839.

(13) Jung, G. S.; Qin, Z.; Buehler, M. J., Molecular mechanics of polycrystalline graphene with enhanced fracture toughness. Extreme Mechanics Letters 2015, 2 (0), 52-59.

(14) He, K.; Poole, C.; Mak, K. F.; Shan, J., Experimental Demonstration of Continuous Electronic Structure Tuning via Strain in Atomically Thin MoS2. Nano Lett. 2013, 13 (6), 2931-2936.

(15) Shen, T.; Penumatcha, A. V.; Appenzeller, J., Strain Engineering for Transition Metal Dichalcogenides Based Field Effect Transistors. ACS Nano 2016, 10 (4), 4712-4718.

(16) Castellanos-Gomez, A.; Roldán, R.; Cappelluti, E.; Buscema, M.; Guinea, F.; van der Zant, H. S. J.; Steele, G. A., Local Strain Engineering in Atomically Thin MoS2. Nano Lett. 2013, 13 (11), 53615366.

(17) Novoselov, K. S.; Geim, A. K.; Morozov, S. V.; Jiang, D.; Zhang, Y.; Dubonos, S. V.; Grigorieva, I. V.; Firsov, A. A., Electric Field Effect in Atomically Thin Carbon Films. Science 2004, 306 (5696), 666.

(18) Lee, C.-H., et al., Atomically thin $\mathrm{p}-\mathrm{n}$ junctions with van der Waals heterointerfaces. Nat Nano 2014, 9 (9), 676-681. 
(19) Kang, K.; Lee, K.-H.; Han, Y.; Gao, H.; Xie, S.; Muller, D. A.; Park, J., Layer-by-layer assembly of two-dimensional materials into wafer-scale heterostructures. Nature $\mathbf{2 0 1 7}$, advance online publication.

(20) Tao, P.; Guo, H.-H.; Yang, T.; Zhang, Z.-D., Stacking stability of MoS 2 bilayer: An ab initio study. Chinese Physics B 2014, 23 (10), 106801.

(21) He, J.; Hummer, K.; Franchini, C., Stacking effects on the electronic and optical properties of bilayer transition metal dichalcogenides MoS2, MoSe2, WS2, and WSe2. Phys. Rev. B 2014, 89 (7), 075409-11.

(22) Kushima, A.; Qian, X.; Zhao, P.; Zhang, S.; Li, J., Ripplocations in van der Waals Layers. Nano Lett. 2015, 15 (2), 1302-1308.

(23) Shanshan, W.; Merce, P.; Harish, B.; Jamie, H. W., Substrate control for large area continuous films of monolayer MoS 2 by atmospheric pressure chemical vapor deposition. Nanotechnology 2016, 27 (8), 085604.

(24) Plimpton, S., Fast Parallel Algorithms for Short-Range Molecular-Dynamics. Journal of Computational Physics 1995, 117 (1), 1-19.

(25) Brenner, D. W.; Shenderova, O. A.; Harrison, J. A.; Stuart, S. J.; Ni, B.; Sinnott, S. B., A secondgeneration reactive empirical bond order (REBO) potential energy expression for hydrocarbons. $J$. Phys.: Condens. Matter 2002, 14 (4), 783-802.

(26) Liang, T.; Phillpot, S. R.; Sinnott, S. B., Parametrization of a reactive many-body potential for Mo-S systems. Phys. Rev. B 2009, 79 (24), 245110-14.

(27) Stewart, J. A.; Spearot, D. E., Atomistic simulations of nanoindentation on the basal plane of crystalline molybdenum disulfide (MoS 2). Modell. Simul. Mater. Sci. Eng. 2013, 21 (4), 045003-15.

(28) Grimme, S., Semiempirical GGA-type density functional constructed with a long-range dispersion correction. J. Comput. Chem. 2006, 27 (15), 1787-1799.

(29) Han, Y.; Li, M.-Y.; Jung, G.-S.; Marsalis, M. A.; Qin, Z.; Buehler, M. J.; Li, L.-J.; Muller, D. A., Sub-nanometre channels embedded in two-dimensional materials. Nat. Mater. 2017.

(30) Tao, P.; Guo, H.-H.; Yang, T.; Zhang, Z.-D., Stacking stability of MoS 2bilayer: An ab initiostudy. Chinese Physics B 2014, 23 (10), 106801-6.

(31) Izrailev, S.; Stepaniants, S.; Isralewitz, B.; Kosztin, D.; Lu, H.; Molnar, F.; Wriggers, W.; Schulten, K., Steered Molecular Dynamics. In Computational Molecular Dynamics: Challenges, Methods, Ideas: Proceedings of the 2nd International Symposium on Algorithms for Macromolecular Modelling, Berlin, May 21-24, 1997, Deuflhard, P.; Hermans, J.; Leimkuhler, B.; Mark, A. E.; Reich, S.; Skeel, R. D., Eds. Springer Berlin Heidelberg: Berlin, Heidelberg, 1999; pp 39-65.

(32) Jung, G. S.; Buehler, M. J., Multiscale Modeling in Muscular-Skeletal Systems. Annu. Rev. Biomed. Eng. 2017, 19 (1). 


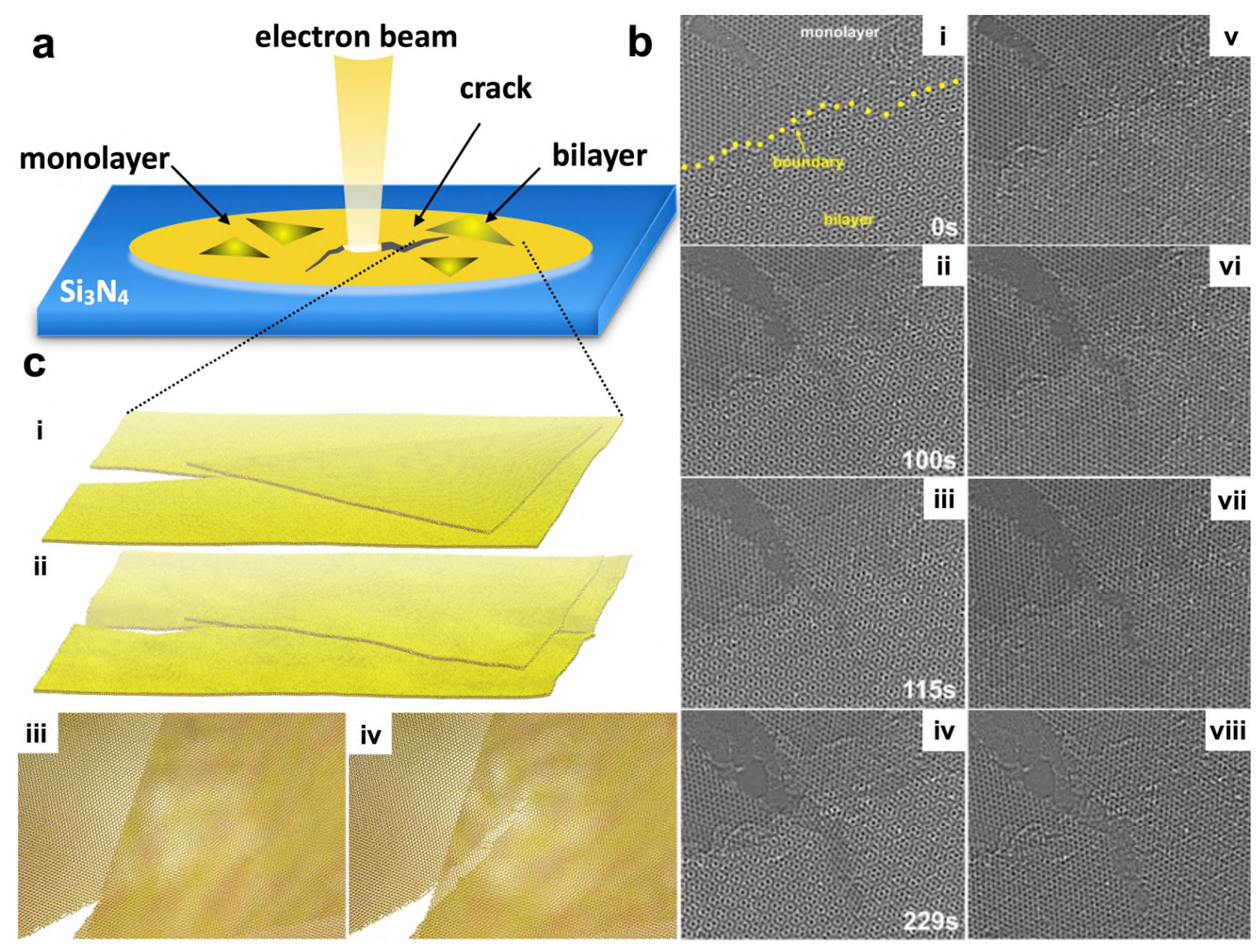

Figure 1. (a) Schematic illustration showing CVD grown mono- and bi-layer $\mathrm{MoS}_{2}$ on $\mathrm{Si}_{3} \mathrm{~N}_{4} \mathrm{TEM}$ grid. The focused e-beam on the suspended layers generates a hole and cracks. Further (b) (A time series of AC-TEM images (i-iv) showing a crack going from the monolayer into the turbostraticallystacked bilayer region. The color of these AC-TEM images is inverted to give a higher visual contrast so that atoms are in white. The crack is restricted in one layer even in the bilayer region. (v-viii) A time series of reconstructed AC-TEM images after filtering out the uncracked layer. (c) The snap shots of MD simulation before (i, iii) and after (ii, iv) crack propagation into the triangular bilayer region. The crack is slightly defected as observed in AC-TEM images. 

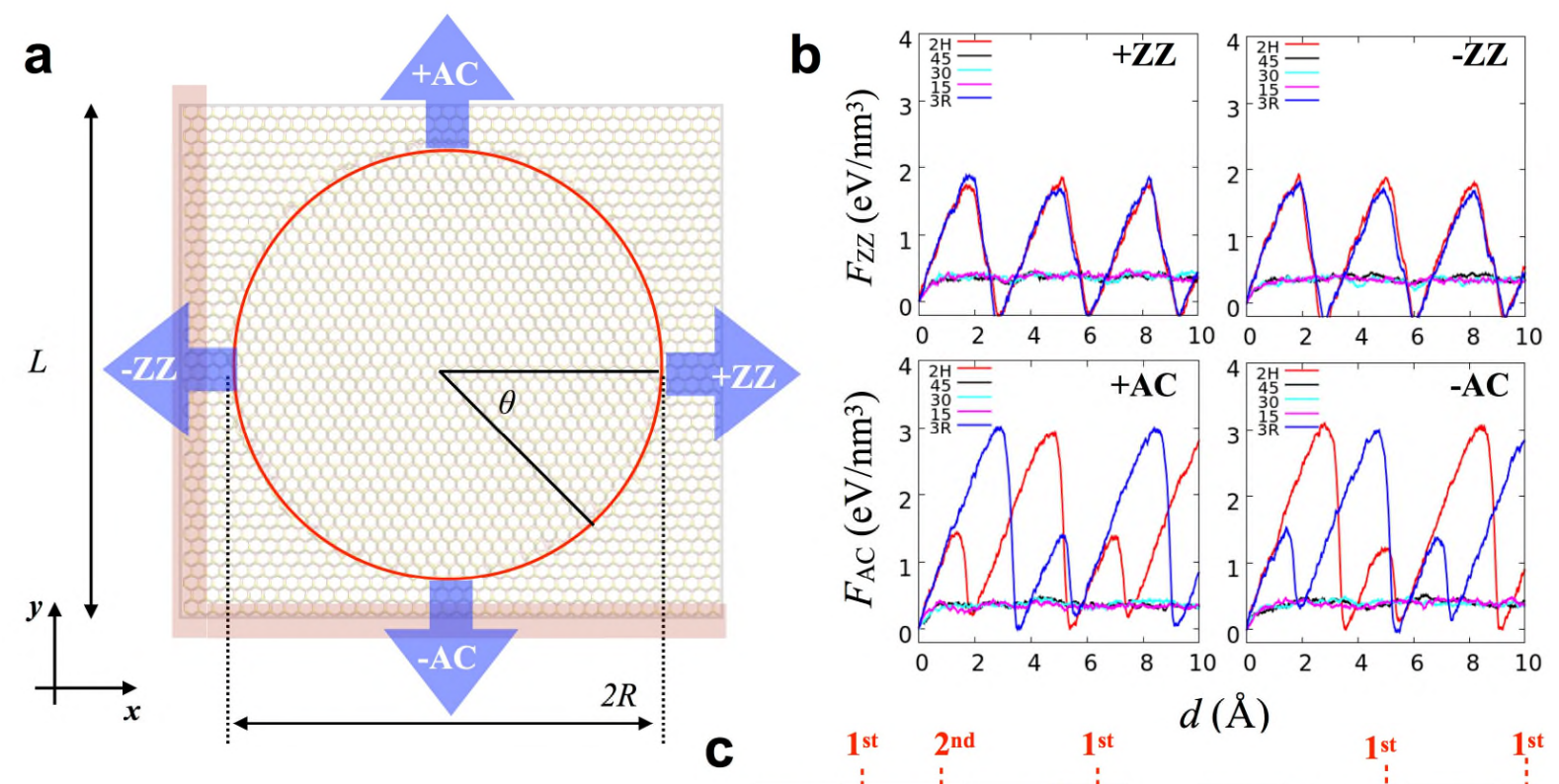

$$
\begin{aligned}
& 2 R=50,70,100,150 \AA \\
& \theta=0^{\circ}(3 \mathrm{R}), 15^{\circ}, 30^{\circ}, 45^{\circ}, 60^{\circ}(2 \mathrm{H}) \\
& L=2 \mathrm{R}+20 \AA
\end{aligned}
$$

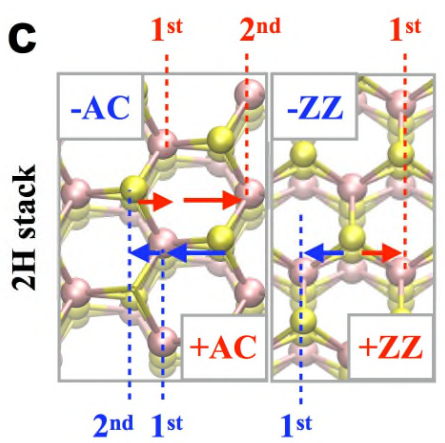

$d(\AA)$

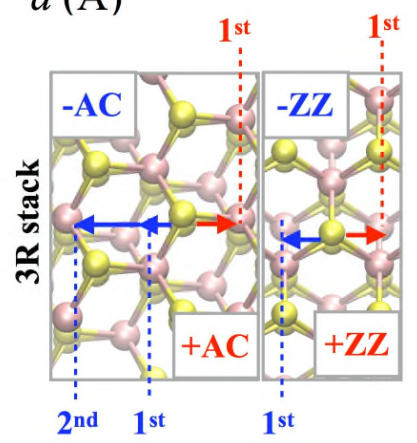

Figure 2. (a) Schematic description of MD simulations for interlayer frictional forces per area $\left(\mathrm{eV} / \mathrm{nm}^{3}\right)$ with four different loading directions: positive and negative zigzag ( $+\mathrm{ZZ}$ and $\left.-\mathrm{ZZ}\right)$ and armchair directions ( $+\mathrm{AC}$ and $-\mathrm{AC}$ ). (b) The forces obtained from the system size of $2 R=15 \mathrm{~nm}(\mathbf{c})$ The relative positions of sulfurs of top and bottom layers. Considering the small difference from the weak van der Waals interactions, the significant difference of friction forces is counterintuitive. The forces and directional dependency can be explained with the relative distance and position of sulfur atoms, suggesting the friction is originated from the geometrical interlocking. The detailed values for all samples are listed in the Table S5 - S8. 

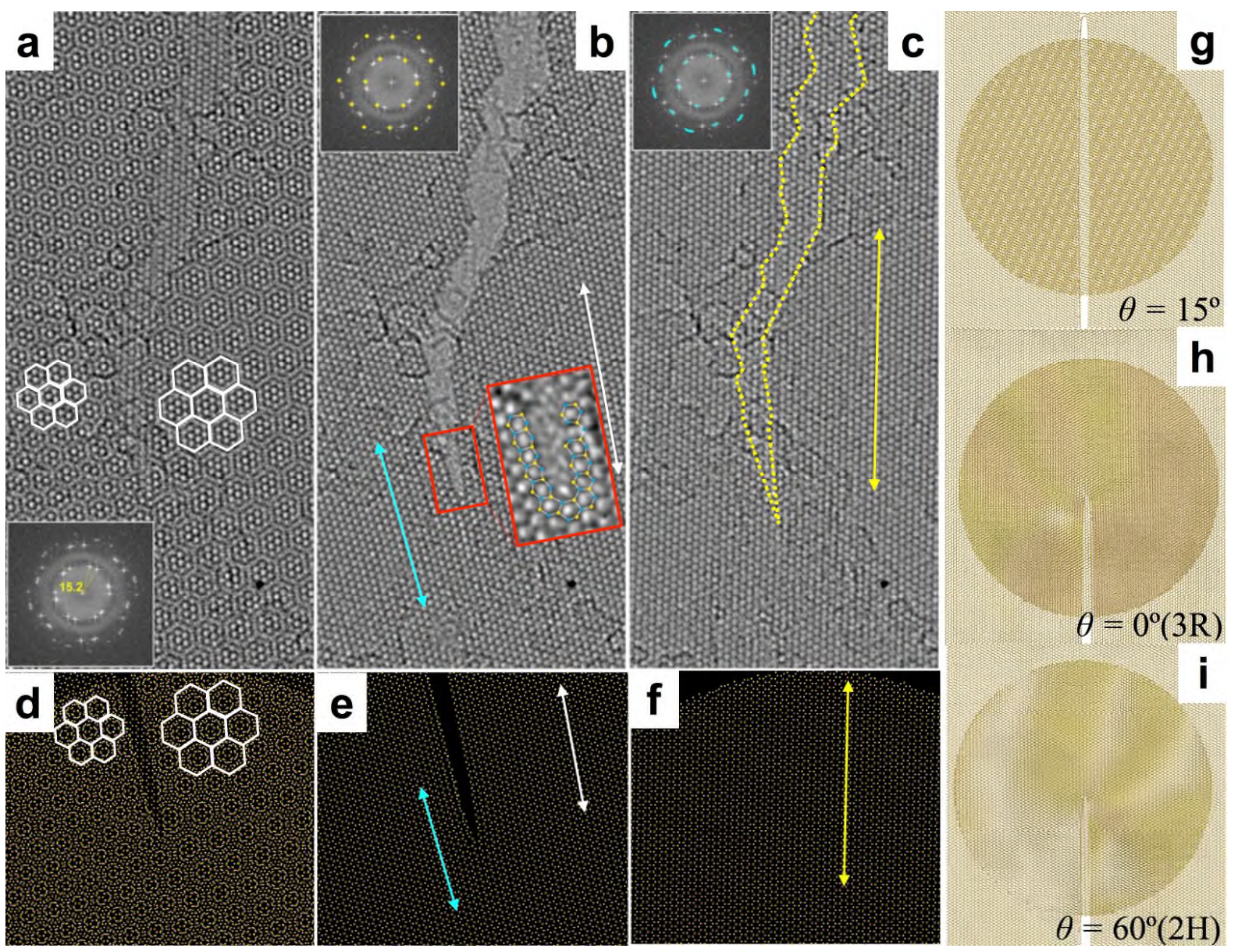

$\theta=60^{\circ}(2 \mathrm{H})$

Figure 3. (a) AC-TEM image showing a crack propagating in one layer of a turbostratically assembled bilayer $\mathrm{MoS}_{2}$. Inset shows the 2D FFT of the AC-TEM image, indicating the twist angle between two layers is around 15 degrees. (b) Reconstructed AC-TEM image after applying a mask to 2D FFT of panel a to remove the lattice contribution from the uncracked layer of $\mathrm{MoS}_{2}$ (as shown the yellow dots of inset at the top left corner). It highlights the lattice configuration of the fractured layer. The crack tip region is zoomed-in with atomic models overlaid, which shows atomic sharpness. The blue and yellow spheres represent Mo and S atoms, respectively. (c) Reconstructed AC-TEM image of the uncracked layer after filtering out the lattice contribution from the fractured layer. The yellow outline indicates the region that has a crack on the other layer. There are no obvious structural changes in this region on the uncracked layer. (d) The MD simulation of crack propagation into finite bilayer region that turbostratically is stacked with 15 degrees. The white hexagons indicate the size of moiré patterns and the asymmetric sizes show good agreement with those in AC-TEM image in panel a. (e) Crack-tip image in the cracked region (f) image of the un-cracked finite layer region. The arrows indicate the zigzag lattice direction in both AC-TEM images and MD. (g-h) The MD results of crack propagation with different stacking, showing clear difference. $2 \mathrm{H}$ and $3 \mathrm{R}$ stack block the crack propagation while the other stacks allow the crack propagate through entire layers (Figure S4 and Supporting Movies 1 and 2). 


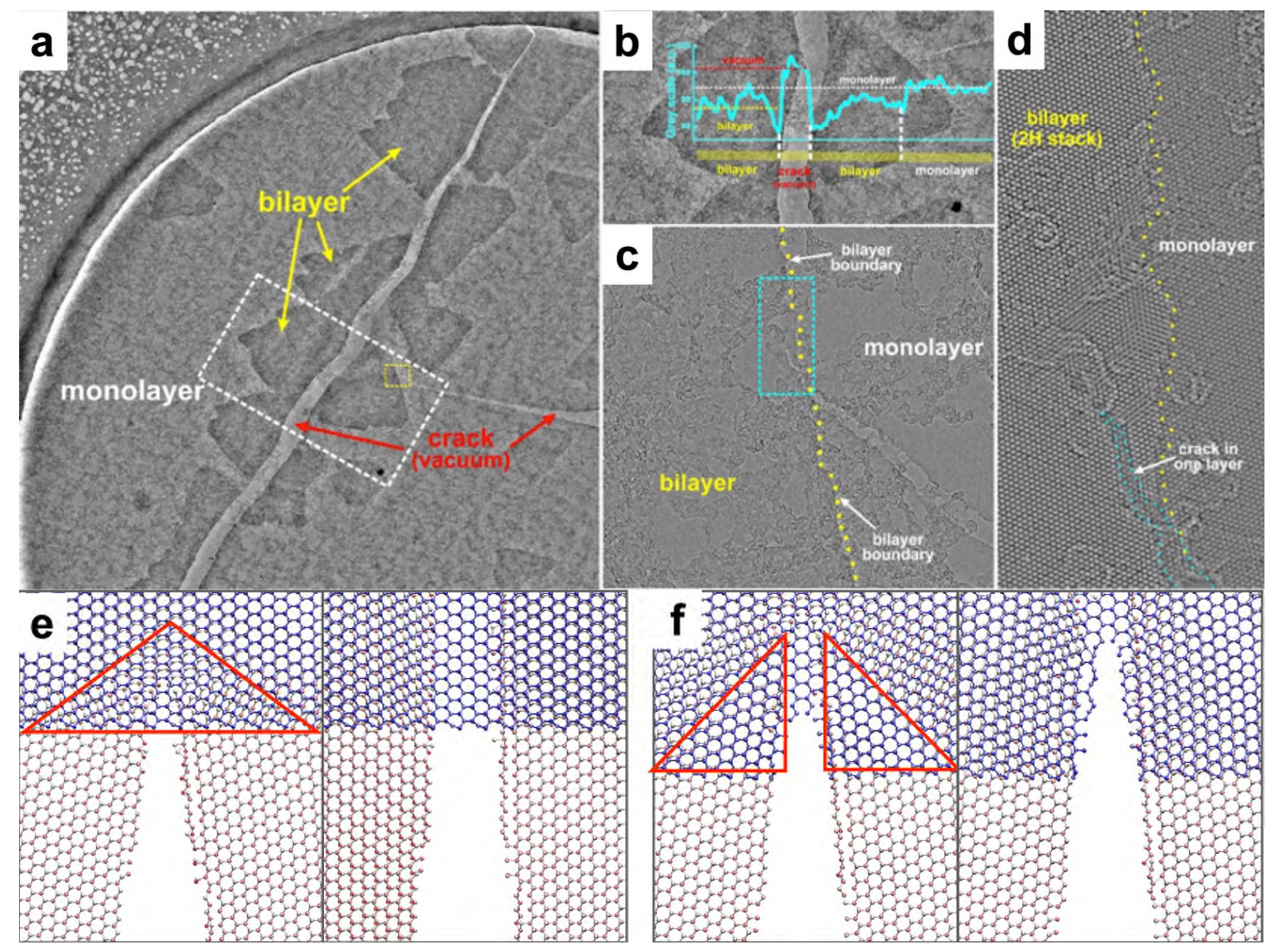

Figure 4. (a) Low-mag TEM image showing two cracks on a suspended $\mathrm{MoS}_{2}$ region. Most areas of this suspended $\mathrm{MoS}_{2}$ region is monolayer, with some small bilayer/multilayer islands distributed on it, as marked by yellow arrow. Two cracks can be observed. The red arrow on the left indicates one of them, and the other one is on the right. (b) Zoom-in TEM image of the region marked by the dashed white box on the left crack in panel a. Boxed intensity line profile along the yellow line is plotted, indicating different layer thickness in this measured region. It also confirms that both two layers fracture in this left crack, leaving vacuum in between. (c) Zoom-in TEM image of the region marked by the dashed yellow box at the tip region on the right crack of panel a. The crack goes from the monolayer $\mathrm{MoS}_{2}$ into the bilayer region. The bilayer boundary is indicated by the yellow dotted line. The crack happens only in one layer in the bilayer region. (d) Zoom-in TEM image of the region in the cyan dashed box in panel $\mathbf{c}$, confirming that the crack is restricted in one layer when it goes into the bilayer region. The bilayer boundary is marked by the yellow dashed line. The bilayer is in the $2 \mathrm{H}$ phase, having the most common $\mathrm{AB}$ stacking mode. In this stacking mode, the top and bottom $\mathrm{MoS}_{2}$ layer are in the same lattice orientation. The S (Mo) atoms of each layer reside on the Mo (S) atoms on the other layer. Therefore, the bilayer region has the same hexagonal lattice pattern as that in the monolayer but having a darker contrast. Since the top and bottom layer are in the same lattice orientation, we cannot use the Fourier transform analysis to split the structure of each layer but can tell the layer thickness change by atomic column contrast. We use this method to tell the crack outline marked by the dashed cyan line. (e) Coherent fracture occurs when both layers move coherently during the crack propagation. The red triangle indicates the regions where the effective tensile stress locally occurs due to the high friction from $2 \mathrm{H}$ stack alignment. The top layer breaks after the crack pass through in the bottom layer. (f) The crack passes through in the bottom layer without breaking the other layer because effective tensile stress does not occur because of distorting lattice from $2 \mathrm{H}$ stack (See Supporting Movies 3 and 4). 

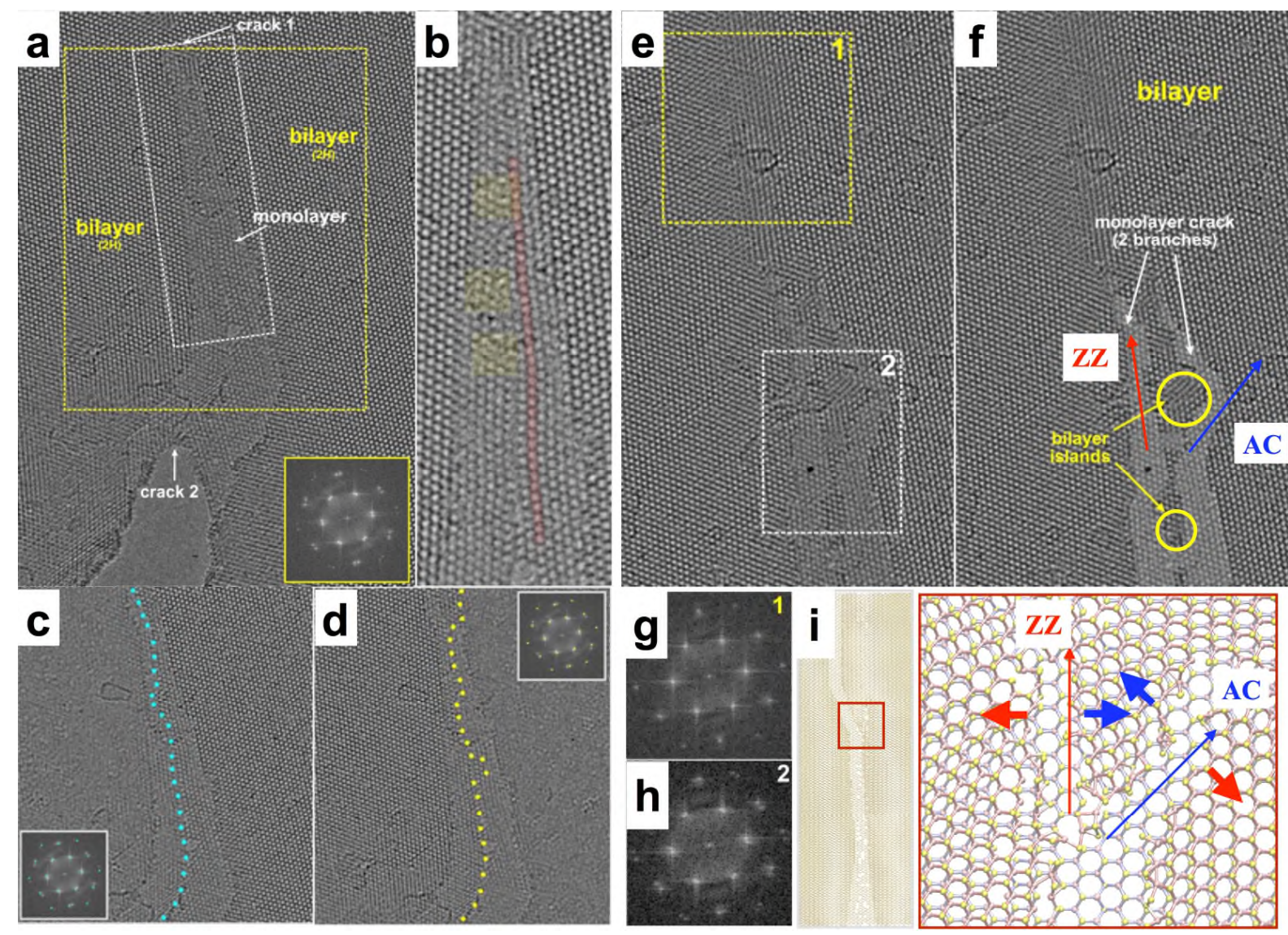

Figure 5. (a) AC-TEM image showing cracks in a $2 \mathrm{H}$-stacked bilayer $\mathrm{MoS}_{2}$ region. Cracks happen in both layers, with a crack in one layer going ahead of the other. Inset shows the 2D FFT of the region in the yellow dashed box. Two sets of FFT spots can be observed. There seems to be some special structure along the midcourt line of the uncracked monolayer region, as lattice shows distortion. (b) Zoom-in AC-TEM image of the region in the white dashed box in panel a, showing that the lattice in the monolayer region is deformed as indicated by the red region. In addition, there are many defects in this region, as marked by the half-transparent rectangles, which might be the reason of lattice distortion. (c) Reconstructed AC-TEM image after applying a mask to one set of the FFT spots, as indicated by the cyan spheres of inset. (d) Reconstructed AC-TEM image after applying a mask to the other set of the FFT spots, as indicated by the yellow spheres of inset. It can be seen that the boundary between the reserved and wiped regions is blurry, and this boundary is not exactly the same in panel $b$ and $\mathrm{c}$, which might be due to the close distance between these two sets of FFT spots. (e) AC-TEM image showing crack 1 in panel a branches off into two cracks, leaving some bilayer islands in between. Two cracks are highlighted by adding half-transparent white masks, as shown in panel (f). 2D FFT analysis are conducted in both the uncracked and cracked regions, marked by the yellow dashed and white dashed boxes in panel e. The FFT patterns 1 and 2 are shown in (g) and (h), respectively. The 2D FFT in the top uncracked region shows only one set of spots, while the FFT in the bottom cracked region shows two set of spots. (i) A crack branches in MD simulations because of defects in uncracked layer with constrained boundary conditions. The red arrows indicate the natural movement due to the external strain, while the blue arrows show the activation of high friction due to the relative configuration of other layers (See Supporting Movies 5 and 6). 